\title{
Modular Hamiltonian of a chiral fermion on the torus
}

\author{
David Blanco $^{*}$ and Guillem Pérez-Nadal ${ }^{\dagger}$ \\ Departamento de Física, FCEN, Universidad de Buenos Aires and IFIBA-CONICET, \\ 1428 Buenos Aires, Argentina
}

(Received 17 May 2019; published 9 July 2019)

\begin{abstract}
We consider a chiral fermion at nonzero temperature on a circle (i.e., on a torus in the Euclidean formalism) and compute the modular Hamiltonian corresponding to a subregion of the circle. We do this by a very simple procedure based on the method of images, which is presumably generalizable to other situations. Our result is nonlocal even for a single interval and even for Neveu-Schwarz boundary conditions. To the best of our knowledge, there are no previous examples of a modular Hamiltonian with this behavior.
\end{abstract}

DOI: 10.1103/PhysRevD.100.025003

\section{INTRODUCTION}

In recent years, the study of entanglement and its measures has proven to be very useful in unveiling some of the deepest properties of quantum field theory (QFT). Entanglement measures are based on the reduced density matrix, or equivalently on (minus) its logarithm, the modular Hamiltonian. Among other applications, the knowledge of modular Hamiltonians was essential for the proof of the averaged null energy condition [1], the derivation of quantum energy inequalities [2,3], and the formulation of a well-defined version of the Bekenstein bound [4]. Modular Hamiltonians also played a key role in applications to holography, the most notable case probably being the derivation of the linearized Einstein equations in the bulk from entanglement properties of the boundary conformal field theory (CFT) [5-8].

There are only few cases where modular Hamiltonians have been computed. The result is universal and local for the vacuum of any QFT reduced to Rindler space $[9,10]$, and from this result one can also derive CFT expressions for the vacuum reduced to a ball in the plane [11], for a thermal state reduced to an interval in the plane in $1+1$ dimensions [12], and for the vacuum reduced to an interval in the cylinder in $1+1$ dimensions [13]. In these cases, the modular Hamiltonian turns out to be local, but nonlocal contributions are expected to appear in general. This was first shown explicitly with the calculation of the modular Hamiltonian of

\footnotetext{
*dblanco@df.uba.ar † guillem@df.uba.ar
}

Published by the American Physical Society under the terms of the Creative Commons Attribution 4.0 International license. Further distribution of this work must maintain attribution to the author(s) and the published article's title, journal citation, and DOI. Funded by SCOAP . the vacuum state reduced to an arbitrary set of disjoint intervals in $1+1$ dimensions for free chiral fermions on the plane [14], later for the cylinder [15], and more recently for free chiral scalars on the plane [16]. Another notable result is the modular Hamiltonian for the vacuum state of any QFT reduced to regions ending on a null plane [17].

In this paper, we compute a new modular Hamiltonian, namely that corresponding to a chiral fermion on the circle at nonzero temperature (i.e., on the torus in Euclidean language). Our analysis is based on the method of images applied to the calculation of the Euclidean propagator, which enables us to map the problem to a similar problem on the plane. The method turns out to be very simple, and we expect it to have applications beyond the case of chiral fermions. Our result is nonlocal even for a single interval, even for Neveu-Schwarz (antiperiodic) boundary conditions.

\section{MODULAR HAMILTONIAN FROM THE RESOLVENT}

Consider a chiral fermion $\psi$ on a circle of length $L$. The Hamiltonian is

$$
H= \pm i \int_{-L / 2}^{L / 2} d x \psi^{\dagger} \psi^{\prime}
$$

where the sign depends on the chirality. Suppose that the field is in a thermal state with inverse temperature $\beta$. The purpose of this paper is to compute the reduced density matrix $\rho_{V}$ corresponding to a subset $V$ of the circle or, equivalently, the modular Hamiltonian

$$
H_{V}=-\log \rho_{V} .
$$

Since the global state is Gaussian, the reduced density matrix is also Gaussian and hence the modular Hamiltonian has the form 


$$
H_{V}=\int_{V} d x d y \psi^{\dagger}(x) K_{V}(x, y) \psi(y) .
$$

As shown in Ref. [18], the kernel $K_{V}$ is related to the twopoint function $G_{V}(x, y)=\left\langle\psi(x) \psi^{\dagger}(y)\right\rangle(x, y \in V)$ by

$$
K_{V}=-\log \left(G_{V}^{-1}-1\right),
$$

where both $K_{V}$ and $G_{V}$ are viewed as operators acting on functions on $V$. This equation can be rewritten as

$$
K_{V}=-\int_{1 / 2}^{\infty} d \xi\left[R_{V}(\xi)+R_{V}(-\xi)\right]
$$

where $R_{V}$ is the resolvent of $G_{V}$

$$
R_{V}(\xi)=\frac{1}{G_{V}+\xi-1 / 2},
$$

as can be easily checked by explicitly performing the integral in Eq. (5). Thus, the problem of computing the modular Hamiltonian reduces to that of finding the resolvent of $G_{V}$.

\section{THE METHOD OF IMAGES}

Our strategy for computing the resolvent is based on the method of images applied to the calculation of the Euclidean propagator $G$, which is defined by

$$
\begin{aligned}
G(x, t ; y, u)= & \theta(t-u)\left\langle e^{H(t-u)} \psi(x) e^{-H(t-u)} \psi^{\dagger}(y)\right\rangle \\
& -\theta(u-t)\left\langle\psi^{\dagger}(y) e^{H(t-u)} \psi(x) e^{-H(t-u)}\right\rangle,
\end{aligned}
$$

for $t-u \in(-\beta, \beta)$ and by analytic continuation for other values of $t$ and $u$, where $\theta$ is the step function. Depending on the spin structure chosen on the circle, the Euclidean propagator can be either periodic or antiperiodic in $x$ with period $L$, and it is antiperiodic in $t$ with period $\beta$. Due to these quasiperiodicity properties, we may view $G$ as a section of a line bundle over a torus of circumferences $L$ and $\beta$. Inserting Eq. (1) in Eq. (7), one sees that the Euclidean propagator satisfies

$$
\left(\partial_{t} \pm i \partial_{x}\right) G=\delta(x-y) \delta(t-u),
$$

for $(x, t)-(y, u) \in(-L, L) \times(-\beta, \beta)$. Identifying $\mathbb{R}^{2}$ with $\mathbb{C}$ via the map $(x, t) \mapsto x \mp i t$, this equation says precisely that $G(z, w)$ is analytic in $z$ for $z \neq w$ and has a simple pole at $z=w$ with residue $\pm 1 /(2 \pi i)$. In other words

$$
G(z, w)= \pm \frac{1}{2 \pi i} \frac{1}{z-w}+F(z, w)
$$

where $F$ is analytic in $z$ for $z-w \in(-L, L) \times(-\beta, \beta)$. In the language of complex variables, the quasiperiodicity conditions read

$$
G\left(z+P_{i}, w\right)=(-1)^{\nu_{i}} G(z, w)
$$

where $\quad P_{1}=L, \quad P_{2}=i \beta, \quad \nu_{1} \in\{0,1\} \quad$ and $\quad \nu_{2}=1$. Equations (9) and (10) have a unique solution. Indeed, the difference $\Delta G$ between two solutions is analytic for $z-w \in(-L, L) \times(-\beta, \beta)$ and satisfies Eq. (10), so it is analytic and bounded throughout the complex plane. By Liouville's theorem, such a function is necessarily a constant, so the antiperiodicity in the imaginary direction implies $\Delta G=0$. In order to find the solution, let us first look at the limiting case $L, \beta \rightarrow \infty$, where the torus becomes a plane and the quasiperiodicity conditions (10) are replaced by the condition that $G$ vanish at infinity. Since the only analytic function that vanishes at infinity is the zero function, the solution of Eq. (9) on the plane is

$$
G(z, w)= \pm \frac{1}{2 \pi i} \frac{1}{z-w} \equiv G_{0}(z, w) .
$$

Going back to the torus, i.e., to generic values of $L$ and $\beta$, we can solve Eqs. (9) and (10) by the method of images

$$
G(z, w)=\sum_{\lambda \in \Lambda}(-1)^{\nu \cdot \lambda} G_{0}(z+\lambda, w),
$$

where $\Lambda$ is the lattice

$$
\Lambda=\left\{\lambda_{1} P_{1}+\lambda_{2} P_{2}, \lambda_{i} \in \mathbb{Z}\right\}
$$

and $\nu \cdot \lambda=\nu_{1} \lambda_{1}+\nu_{2} \lambda_{2}$. Indeed, the function (12) clearly has the form (9), and one can easily check that it satisfies the quasiperiodicity conditions (10).

Let now $x, y \in V$. It is clear from Eq. (7) that $G_{V}(x, y)=$ $G\left(x, 0^{+} ; y, 0\right)$ (note that it is important to take the limit $t \rightarrow 0$ from above, because if we take it from below we pick a delta function). With our identification of $\mathbb{R}^{2}$ with $\mathbb{C}$, we thus have

$$
G_{V}(x, y)=G(x \mp i \epsilon, y)
$$

so, by Eq. (12)

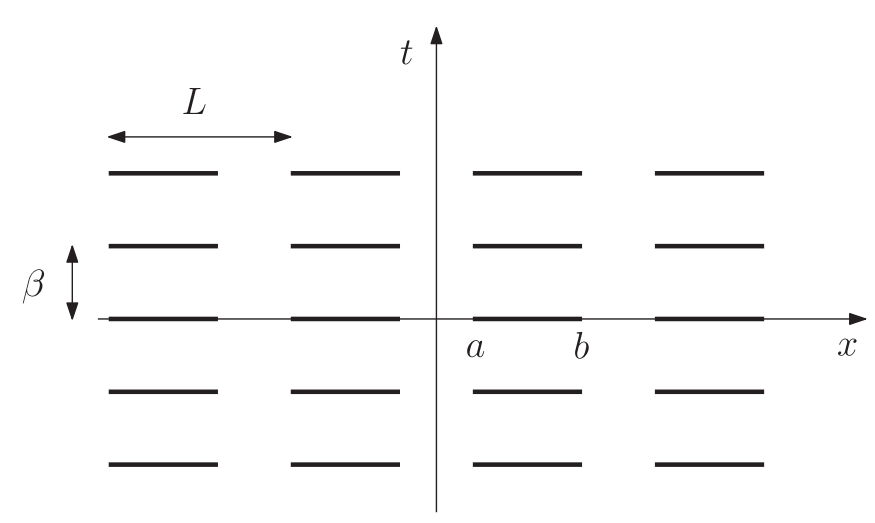

FIG. 1. The region $V_{\Lambda}$ when $V$ is the interval $(a, b)$. 


$$
G_{V}(x, y)=\sum_{\lambda \in \Lambda}(-1)^{\nu \cdot \lambda} G_{0 V_{\Lambda}}(x+\lambda, y),
$$

$$
\left(G_{V}^{n}\right)(x, y)=\sum_{\lambda \in \Lambda}(-1)^{\nu \cdot \lambda}\left(G_{0 V_{\Lambda}}^{n}\right)(x+\lambda, y),
$$

where $V_{\Lambda}=\bigcup_{\lambda \in \Lambda}(V+\lambda)$; see Fig. 1, and

$$
G_{0 V_{\Lambda}}(u, v)=G_{0}(u \mp i \epsilon, v),
$$

for $u, v \in V_{\Lambda}$. The main reason why the method of images is useful for us is that Eq. (15) also holds for the powers of the operators involved for any $n \in \mathbb{N}$, where $\left(A^{n}\right)(u, v)$ denotes the kernel of the operator $A^{n}$ (not to be confused with $[A(u, v)]^{n}$ ). We can see this by induction. First, the above equation is satisfied for $n=1$ [this is Eq. (15)]. And second, if it holds for some $n \in \mathbb{N}$ we have

$$
\begin{aligned}
\left(G_{V}^{n+1}\right)(x, y) & =\int_{V} d z G_{V}(x, z)\left(G_{V}^{n}\right)(z, y) \\
& =\sum_{\lambda, \mu \in \Lambda}(-1)^{\nu \cdot(\lambda+\mu)} \int_{V} d z G_{0 V_{\Lambda}}(x+\lambda, z)\left(G_{0 V_{\Lambda}}^{n}\right)(z+\mu, y) \\
& =\sum_{\lambda, \mu \in \Lambda}(-1)^{\nu \cdot(\lambda+\mu)} \int_{V} d z G_{0 V_{\Lambda}}(x+\lambda+\mu, z+\mu)\left(G_{0 V_{\Lambda}}^{n}\right)(z+\mu, y) \\
& =\sum_{\lambda^{\prime}, \mu \in \Lambda}(-1)^{\nu \cdot \lambda^{\prime}} \int_{V+\mu} d z^{\prime} G_{0 V_{\Lambda}}\left(x+\lambda^{\prime}, z^{\prime}\right)\left(G_{0 V_{\Lambda}}^{n}\right)\left(z^{\prime}, y\right) \\
& =\sum_{\lambda^{\prime} \in \Lambda}(-1)^{\nu \cdot \lambda^{\prime}} \int_{V_{\Lambda}} d z^{\prime} G_{0 V_{\Lambda}}\left(x+\lambda^{\prime}, z^{\prime}\right)\left(G_{0 V_{\Lambda}}^{n}\right)\left(z^{\prime}, y\right) \\
& =\sum_{\lambda^{\prime} \in \Lambda}(-1)^{\nu \cdot \lambda^{\prime}}\left(G_{0 V_{\Lambda}}^{n+1}\right)\left(x+\lambda^{\prime}, y\right) .
\end{aligned}
$$

In the third equality we have used the translational invariance of $G_{0}$, and in the fourth we have defined $\lambda^{\prime}=\lambda+\mu$ and $z^{\prime}=z+\mu$. Equation (17) implies that the method of images works for any function of $G_{V}$ which can be expressed as a power series. In particular, it works for the resolvent

$$
R_{V}(\xi ; x, y)=\sum_{\lambda \in \Lambda}(-1)^{\nu \cdot \lambda} R_{0 V_{\Lambda}}(\xi ; x+\lambda, y) .
$$

In the case of zero temperature, $\beta \rightarrow \infty$, the terms with $\lambda_{2} \neq 0$ do not contribute to the sum (12), so the lattice $\Lambda$ effectively reduces to $\{m L, m \in \mathbb{Z}\}$ and, in consequence, the region $V_{\Lambda}$ reduces to an arrangement of segments in the real line. The resolvent $R_{0 V_{\Lambda}}$ is well known in that case [14], so we can use it to obtain $R_{V}$ via the above equation. To the best of our knowledge, $R_{0 V_{\Lambda}}$ is not known for generic temperatures, where $V_{\Lambda}$ is a collection of segments distributed all over the complex plane, but it can be easily computed as we will explain in the next section.

\section{THE RESOLVENT FOR A GENERIC SET OF SEGMENTS IN THE PLANE}

Consider a curve $\gamma$ in the complex plane with both end points at infinity and a subset $A \subset \gamma$; see Fig. 2. As shown in the figure, $\gamma$ divides the plane into two regions: the one to the left of the curve ( + region) and the one to the right ( - region); if $\gamma$ is the real line these regions are the upper and lower half-planes, respectively. The purpose of this section is to compute the resolvent of the operator $G_{0 A}$ with kernel

$$
G_{0 A}(u, v)=G_{0}\left(u^{\mp}, v\right)= \pm \frac{1}{2 \pi i} \frac{1}{u^{\mp}-v},
$$

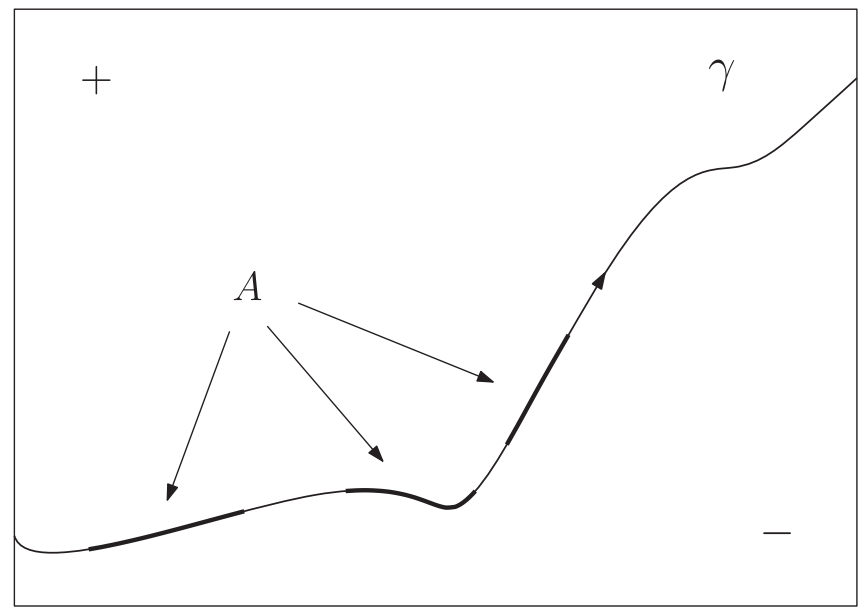

FIG. 2. A curve $\gamma$ in the complex plane and a subset $A \subset \gamma$. The $+/-$ region is the region to the left/right of the curve. 
for $u, v \in A$, where $F\left(u^{\mp}\right)$ denotes the limit of $F$ as $u$ is approached from the $\mp$ region. This resolvent is known in the case where $\gamma$ is the real line [14]; as we will see, the computation for $\gamma$ generic is remarkably simple.

We will first obtain an expression for the powers of $G_{0 A}$. Then we will insert that expression into the expansion of the resolvent in powers of $G_{0 A}$ and find that it is easy to perform the sum. For the square, we have

$$
\begin{aligned}
\left(G_{0 A}^{2}\right)(u, v) & =\int_{A} d w G_{0 A}(u, w) G_{0 A}(w, v) \\
& =\frac{1}{(2 \pi i)^{2}} \int_{A} \frac{d w}{\left(u^{\mp}-w\right)\left(w-v^{ \pm}\right)} \\
& =\frac{1}{2 \pi i} G_{0 A}(u, v)\left[\omega_{A}^{ \pm}(u)+\omega_{A}^{\mp}(v)\right],
\end{aligned}
$$

where

$$
\omega_{A}^{ \pm}(u)= \pm \int_{A} \frac{d v}{u^{\mp}-v} .
$$

Note that

$$
\omega_{A}^{+}+\omega_{A}^{-}=2 \pi i .
$$

Indeed, if $A^{ \pm}$is a slight deformation of $A$ which has the same end points but travels through the \pm region, we have

$$
\begin{aligned}
\omega_{A}^{+}(u)+\omega_{A}^{-}(u) & =\int_{A} \frac{d v}{u^{-}-v}-\int_{A} \frac{d v}{u^{+}-v} \\
& =\int_{A^{+}} \frac{d v}{u-v}-\int_{A^{-}} \frac{d v}{u-v} \\
& =\oint \frac{d v}{u-v}=2 \pi i,
\end{aligned}
$$

where the contour in the last integral encircles $A$, and hence $u$. We can rewrite Eq. (21) as an operator equation

$$
G_{0 A}^{2}=\frac{1}{2 \pi i}\left(\omega_{A}^{ \pm} G_{0 A}+G_{0 A} \omega_{A}^{\mp}\right) .
$$

Now, using Eqs. (23) and (25) it is a simple matter to check that the operator-valued function

$$
\bar{G}_{0 A}(s)=(1+s)^{-\omega_{A}^{ \pm} /(2 \pi i)} G_{0 A}(1+s)^{-\omega_{A}^{\mp} /(2 \pi i)}
$$

satisfies $\bar{G}_{0 A}^{\prime}=-\bar{G}_{0 A}^{2}$. In turn, this implies for the $n$th derivative $\bar{G}_{0 A}^{(n)}=(-1)^{n} n ! \bar{G}_{0 A}^{n+1}$, as can be easily shown by induction. Noting that $\bar{G}_{0 A}(0)=G_{0 A}$, we thus obtain

$$
G_{0 A}^{n+1}=\frac{(-1)^{n}}{n !} \bar{G}_{0 A}^{(n)}(0) .
$$

Inserting this expression into the expansion of the resolvent in powers of $G_{0 A}$ (which is a geometric series), we recognize the Taylor series of $\bar{G}_{0 A}$

$$
\begin{aligned}
R_{0 A}(\xi) & =\frac{1}{G_{0 A}+\xi-1 / 2} \\
& =\frac{1}{\xi-1 / 2}\left[1-\frac{1}{\xi-1 / 2} \sum_{n=0}^{\infty} \frac{(-1)^{n}}{(\xi-1 / 2)^{n}} G_{0 A}^{n+1}\right] \\
& =\frac{1}{\xi-1 / 2}\left[1-\frac{\bar{G}_{0 A}(1 /(\xi-1 / 2))}{\xi-1 / 2}\right] \\
& =\frac{1}{\xi-1 / 2}\left[1-\frac{e^{-i k(\xi) \omega_{A}^{ \pm}} G_{0 A} e^{i k(\xi) \omega_{A}^{ \pm}}}{\xi+1 / 2}\right],
\end{aligned}
$$

where in the last step we have used (23) and defined

$$
k(\xi)=\frac{1}{2 \pi} \log \frac{\xi-1 / 2}{\xi+1 / 2} .
$$

Equation (28) gives the resolvent for a generic subset $A$ of a generic curve. Let us particularize it to the case where $A$ is a collection of horizontal segments, $A=\bigcup_{\alpha}\left[\left(a_{\alpha}, b_{\alpha}\right)+i \eta_{\alpha}\right]$ with $a_{\alpha}, b_{\alpha}, \eta_{\alpha} \in \mathbb{R}$. In this case, the integral (22) is easily computed

$$
\omega_{A}^{ \pm}(u)= \pm \sum_{\alpha} \log \frac{a_{\alpha}+i \eta_{\alpha}-u \pm i \epsilon}{b_{\alpha}+i \eta_{\alpha}-u \pm i \epsilon},
$$

and, using the relation $1 /(x \mp i \epsilon)=1 / x \pm i \pi \delta(x)$ (there is a principal part implicit in the first term), the resolvent (28) takes the form

$$
R_{0 A}(\xi ; u, v)=\frac{1}{\xi^{2}-1 / 4}\left\{\xi \delta(u-v) \mp \frac{e^{-i k(\xi)\left[\omega_{A}^{ \pm}(u)-\omega_{A}^{ \pm}(v)\right]}}{2 \pi i(u-v)}\right\}
$$

This result agrees with that of Eq. [14] in the case where $A$ is contained in the real line.

\section{MODULAR HAMILTONIAN ON THE TORUS}

Our last step is to use the resolvent just computed to obtain the resolvent on the torus by the method of images, Eq. (19), and from it the modular Hamiltonian. For simplicity, we concentrate on the case where $V$ is a single interval, $V=(a, b)$, but the analysis that follows extends straightforwardly to the general case of multiple intervals. Setting $A=V_{\Lambda}$ in (30) yields

$$
\omega_{V_{\Lambda}}^{ \pm}(u)= \pm \sum_{\lambda \in \Lambda} \log \frac{a-u+\lambda \pm i \epsilon}{b-u+\lambda \pm i \epsilon} .
$$

This series is ambiguous because it is not absolutely convergent. However, its second derivative is unambiguous, 


$$
\begin{aligned}
\left(\omega_{V_{\Lambda}}^{ \pm}\right)^{\prime \prime}(u) & =\mp \sum_{\lambda \in \Lambda}\left[\frac{1}{(a-u+\lambda \pm i \epsilon)^{2}}-\frac{1}{(b-u+\lambda \pm i \epsilon)^{2}}\right] \\
& =\mp[\wp(a-u \pm i \epsilon)-\wp(b-u \pm i \epsilon)],
\end{aligned}
$$

where $\wp$ is the Weierstrass elliptic function (see Ref. [19] for a review). Since the latter is related to the Weierstrass sigma function

$$
\sigma(z)=z \prod_{\lambda \neq 0}\left(1+\frac{z}{\lambda}\right) e^{-\frac{z}{\lambda}+\frac{1}{2}\left(\frac{z}{\lambda}\right)^{2}},
$$

by $\wp=-(\log \sigma)^{\prime \prime}$, we conclude that

$$
\omega_{V_{\Lambda}}^{ \pm}(u)=\omega^{ \pm}(u)+c^{ \pm} u+d^{ \pm},
$$

where

$$
\omega^{ \pm}(u)= \pm \log \frac{\sigma(a-u \pm i \epsilon)}{\sigma(b-u \pm i \epsilon)},
$$

and $c^{ \pm}, d^{ \pm}$are undetermined constants. The Weierstrass sigma function is quasiperiodic,

$$
\sigma(z+\lambda)=(-1)^{\lambda_{1}+\lambda_{2}+\lambda_{1} \lambda_{2}} e^{\lambda \cdot \zeta(P / 2)(2 z+\lambda)} \sigma(z),
$$

where $\zeta=(\log \sigma)^{\prime}$ and $\lambda \cdot \zeta(P / 2)=\lambda_{1} \zeta\left(P_{1} / 2\right)+\lambda_{2} \zeta\left(P_{2} / 2\right)$. Therefore, $\omega^{ \pm}$is also quasiperiodic,

$$
\omega^{ \pm}(u+\lambda)=\omega^{ \pm}(u) \pm 2 l \lambda \cdot \zeta(P / 2),
$$

where $l=b-a$. Substituting the resolvent (31) with $A=V_{\Lambda}$ into Eq. (19), and using Eqs. (35), (36), and (38), we obtain for the resolvent on the torus

$R_{V}(\xi ; x, y)=\frac{1}{\xi^{2}-1 / 4}\left[\xi \delta(x-y)-\frac{e^{\mp i k(\xi) \Delta \omega(x, y)}}{2 \pi i} F^{ \pm}(\xi ; x, y)\right]$,

where

$$
\Delta \omega(x, y)=\log \frac{\sigma(a-x) \sigma(b-y)}{\sigma(b-x) \sigma(a-y)},
$$

which does not need the regulator $\epsilon$ because the argument of the logarithm is real and positive, and

$$
\begin{aligned}
F^{ \pm}(\xi ; z, w)= & \pm \sum_{\lambda \in \Lambda}(-1)^{\nu \cdot \lambda} e^{\mp 2 i k(\xi) l \lambda \cdot \zeta(P / 2)} \\
& \times \frac{e^{-i k(\xi) c^{ \pm}(z+\lambda-w)}}{z+\lambda-w} .
\end{aligned}
$$

Note that the constant $d^{ \pm}$has dropped out because $R_{0 V_{\Lambda}}$ only involves the difference $\omega_{V_{\Lambda}}^{ \pm}(u)-\omega_{V_{\Lambda}}^{ \pm}(v)$; see Eq. (31). Note also that the summand above may diverge exponentially as $\lambda$ grows; preventing this divergence fixes $c^{ \pm}=\mp 2 l \zeta\left(P_{2} / 2\right) / P_{2}$. Therefore, the ambiguity in $\omega_{V_{\Lambda}}^{ \pm}$ disappears from the resolvent on the torus. Now, $F^{ \pm}$has the following properties: (i) it is analytic in $z$ for $z-w \in$ $(-L, L) \times(-\beta, \beta)$ except for a simple pole at $z=w$ with residue \pm 1 , and (ii) it is quasiperiodic

$F^{ \pm}\left(\xi ; z+P_{i}, w\right)=(-1)^{\nu_{i}} e^{ \pm 2 i k(\xi) l \zeta\left(P_{i} / 2\right)} F^{ \pm}(\xi ; z, w)$.

By the argument we gave under Eq. (10), there is only one function with these properties. This function is

$$
F^{ \pm}(\xi ; z, w)= \pm \frac{1}{\sigma(z-w)} \frac{\sigma_{\nu}(z-w \pm i k(\xi) l)}{\sigma_{\nu}( \pm i k(\xi) l)},
$$

where

$\sigma_{\nu}(z)=e^{-\left[\nu_{1} \zeta\left(P_{2} / 2\right)+\zeta\left(P_{1} / 2\right)\right] z} \sigma\left(z+\nu_{1} P_{2} / 2+P_{1} / 2\right)$

(recall that $\nu_{2}=1$ ). Indeed, $\sigma$ is analytic, has a simple zero at the origin with $\sigma^{\prime}(0)=1$, and does not vanish anywhere else in the region $(-L, L) \times(-\beta, \beta)$. Together with quasiperiodicity, this implies that the second ratio in Eq. (43) is analytic, from which property (i) follows. On the other hand, Eq. (37) and the relation $P_{2} \zeta\left(P_{1} / 2\right)-P_{1} \zeta\left(P_{2}\right)=i \pi$ imply

$$
\sigma_{\nu}\left(z+P_{i}\right)=(-1)^{\nu_{i}+1} e^{\zeta\left(P_{i} / 2\right)\left(2 z+P_{i}\right)} \sigma_{\nu}(z),
$$

from which property (ii) follows. Thus, Eqs. (39) and (43) give the resolvent on the torus. Inserting it into Eq. (5), noting that the terms with a delta function cancel and changing the variable of integration from $\xi$ to $k(\xi)$ yields the modular Hamiltonian

$$
\begin{aligned}
& K_{V}(x, y)=\frac{\mp i}{\sigma(x-y)} \int_{-\infty}^{\infty} d k f(k ; x, y) \\
& f(k ; x, y)=e^{-i k \Delta \omega(x, y)} \frac{\sigma_{\nu}(x-y+i k l)}{\sigma_{\nu}(i k l)} .
\end{aligned}
$$

We have checked that this result coincides with known results in the limits $L \rightarrow \infty$ [12] and $\beta \rightarrow \infty$ [15], including the presence of a nonlocal term in the case $\nu_{1}=0$ (periodic, or Ramond, boundary conditions). In order to obtain a more explicit expression for $L$ and $\beta$ generic, note from Eq. (45) that $f$ is quasiperiodic in $k$,

$$
f(k+\beta / l ; x, y)=e^{-i\left\{\frac{\beta}{T} \Delta \omega(x, y)-2 \eta(x-y)\right\}} f(k ; x, y),
$$

where $\eta=-i \zeta(i \beta / 2)\left[\zeta\right.$ is odd and satisfies $\zeta\left(z^{*}\right)=\zeta^{*}(z)$, so $\eta$ is real]. Therefore,

$$
\int_{-\infty}^{\infty} d k f(k ; x, y)=I(x, y) \int_{-\frac{\beta}{2 l}}^{\frac{\beta}{2 l}} d k f(k ; x, y),
$$

where 


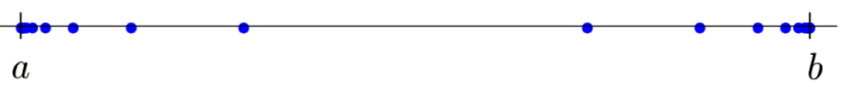

FIG. 3. The first few points that the nonlocal term (52) of the modular Hamiltonian couples to the midpoint of the interval, for $l / L=0.6$ and $l / \beta=0.12$. They tend to accumulate near the end points.

$$
\begin{aligned}
I(x, y) & =\sum_{n \in \mathbb{Z}} e^{-\mathrm{in}\left\{\frac{\beta}{l} \Delta \omega(x, y)-2 \eta(x-y)\right\}} \\
& =2 \pi \sum_{n \in \mathbb{Z}} \delta\left(\frac{\beta}{l} \Delta \omega(x, y)-2 \eta(x-y)+2 \pi n\right) .
\end{aligned}
$$

Now, for $x$ fixed, the argument of the delta function above decreases monotonically from $\infty$ to $-\infty$ as $y$ goes from $a$ to $b$, and hence it has a unique zero for each $n$. For $n=0$, this is $y=x$. Noting that $f(k ; x, x)=1$ one easily obtains the local contribution to the modular Hamiltonian

$K_{V}^{\mathrm{loc}}(x, y)= \pm i\left[\tilde{\beta}(x) \delta^{\prime}(x-y)+\frac{1}{2} \tilde{\beta}^{\prime}(x) \delta(x-y)\right]$,

where

$$
\tilde{\beta}(x)=\frac{2 \pi}{\zeta(b-x)-\zeta(a-x)-2 \eta l / \beta} .
$$

In order to obtain the nonlocal contribution (i.e., the contribution from the remaining values of $n$ ), note that the delta function in Eq. (49) selects precisely those pairs of points $(x, y)$ for which $f$ is periodic. On the other hand, $f$ satisfies a second quasiperiodicity property, analogous to Eq. (47) with $\beta$ replaced by $i L$ and $\eta$ replaced by $i \zeta(L / 2)$. Using these two facts, one can rewrite the integral on the right-hand side of Eq. (48) in terms of a contour integral encircling a single pole. Evaluating the latter by residues finally yields the nonlocal contribution to the modular Hamiltonian

$$
\begin{aligned}
K_{V}^{\text {non-loc }}(x, y)= & \frac{ \pm 2 i \pi^{2}}{l \sinh \left(\frac{L}{2 l} \Delta \omega(x, y)-\zeta(L / 2)(x-y)\right)} \\
& \times \sum_{n \neq 0}(-1)^{\nu_{1} n} \delta\left(\frac{\beta}{l} \Delta \omega(x, y)\right. \\
& -2 \eta(x-y)+2 \pi n) .
\end{aligned}
$$

For $x$ fixed, this nonlocal term has support in an infinite number of points which tend to accumulate near the end points of the interval, as shown in Fig. 3. Thus, the modular Hamiltonian is highly nonlocal, both for Ramond $\left(\nu_{1}=0\right)$ and Neveu-Schwarz $\left(\nu_{1}=1\right)$ boundary conditions.

The above is a novel explicit example of a modular Hamiltonian, which has the interesting property of being highly nonlocal even for a single interval, for any choice of boundary conditions. It thus opens a new window for the exploration of the entanglement properties of QFT.

\section{ACKNOWLEDGMENTS}

The authors would like to thank Horacio Casini, Pascal Fries, Alan Garbarz, Gaston Giribet, Andrés Goya, Martín Mereb, and Ignacio Reyes for very fruitful discussions. This work was supported by CONICET and Universidad de Buenos Aires, Argentina.

Note added.-While this manuscript was nearing completion a work with related results appeared [20], and another similar study [21] appeared after this paper was first announced on arXiv. We leave the comparison between the three analyses for future work.
[1] T. Faulkner, R. G. Leigh, O. Parrikar, and H. Wang, Modular Hamiltonians for deformed half-spaces and the averaged null energy condition, J. High Energy Phys. 09 (2016) 038.

[2] D. D. Blanco and H. Casini, Localization of Negative Energy and the Bekenstein Bound, Phys. Rev. Lett. 111, 221601 (2013).

[3] D. Blanco, H. Casini, M. Leston, and F. Rosso, Modular energy inequalities from relative entropy, J. High Energy Phys. 01 (2018) 154.

[4] H. Casini, Relative entropy and the Bekenstein bound, Classical Quantum Gravity 25, 205021 (2008).

[5] T. Faulkner, M. Guica, T. Hartman, R. C. Myers, and M. Van Raamsdonk, Gravitation from entanglement in holographic CFTs, J. High Energy Phys. 03 (2014) 051.
[6] N. Lashkari, M. B. McDermott, and M. Van Raamsdonk, Gravitational dynamics from entanglement 'thermodynamics,' J. High Energy Phys. 04 (2014) 195.

[7] D. Blanco, M. Leston, and G. Pérez-Nadal, Gravity from entanglement for boundary subregions, J. High Energy Phys. 06 (2018) 130.

[8] B. Swingle and M. Van Raamsdonk, Universality of gravity from entanglement, arXiv:1405.2933.

[9] W. G. Unruh, Notes on black hole evaporation, Phys. Rev. D 14, 870 (1976).

[10] J. J. Bisognano and E. H. Wichmann, On the duality condition for quantum fields, J. Math. Phys. (N.Y.) 17, 303 (1976).

[11] H. Casini, M. Huerta, and R. C. Myers, Towards a derivation of holographic entanglement entropy, J. High Energy Phys. 05 (2011) 036. 
[12] T. Hartman and N. Afkhami-Jeddi, Speed limits for entanglement, arXiv:1512.02695.

[13] J. Cardy and E. Tonni, Entanglement Hamiltonians in twodimensional conformal field theory, J. Stat. Mech. (2016) 123103.

[14] H. Casini and M. Huerta, Reduced density matrix and internal dynamics for multicomponent regions, Classical Quantum Gravity 26, 185005 (2009).

[15] I. Klich, D. Vaman, and G. Wong, Entanglement Hamiltonians for Chiral Fermions with Zero Modes, Phys. Rev. Lett. 119, 120401 (2017).

[16] R. E. Arias, H. Casini, M. Huerta, and D. Pontello, Entropy and modular Hamiltonian for a free chiral scalar in two intervals, Phys. Rev. D 98, 125008 (2018).
[17] H. Casini, E. Teste, and G. Torroba, Modular Hamiltonians on the null plane and the Markov property of the vacuum state, J. Phys. A 50, 364001 (2017).

[18] I. Peschel, Calculation of reduced density matrices from correlation functions, J. Phys. A 36, L205 (2003).

[19] K. Chandrasekharan, Elliptic Functions, Grundlehren der Mathematischen Wissenschaften, A Series of Comprehensive Studies in Mathematics Vol. 281 (Springer, Berlin, Heidelberg, 1985).

[20] S. Hollands, On the modular operator of mutli-component regions in chiral CFT, arXiv:1904.08201.

[21] P. Fries and I. A. Reyes, The entanglement spectrum of chiral fermions on the torus, arXiv:1905.05768. 